\title{
Activity evaluation of some psychoactive drugs with the application of QSAR/QSPR modeling methods
}

\author{
Piotr Kawczak $\mathbb{D}^{1} \cdot$ Leszek Bober ${ }^{2} \cdot$ Tomasz Bączek $^{1,3}$
}

Received: 16 April 2018 / Accepted: 2 August 2018 / Published online: 10 August 2018

(c) The Author(s) 2018

\begin{abstract}
A set of psychoactive drugs has been analyzed with the use of quantitative structure-activity/property relationships methods. The purpose of this study was to demonstrate both the common and differentiating characteristics of the above-mentioned chemical compounds, physicochemical as well as pharmacological based on the quantum chemical calculations and selected biological activity data and chromatographic retention parameters. During the study, the ab initio model of molecular modeling was performed and PCA, FA, and MLR as the types of chemometric approach. QSAR/QSPR models were proposed based on chosen statistically significant descriptors. The relationship between the structure and biological activity data was able to class and describe the psychoactive properties of the molecules studied. The applied chemometric approaches revealed the influential features of tested structures responsible for their pharmacological activity together with some additional physicochemical properties.
\end{abstract}

Keywords Psychoactive drugs $\cdot$ Molecular modeling $\cdot$ Structural analysis $\cdot$ Descriptors $\cdot$ QSAR; QSPR

\section{Introduction}

The term psychoactive/psychotropic drug is defined as the collective name of substances whose only or main action is the effect on mental activities. In general, they are agents that act on the central nervous system, but there are also psychotropic agents in medicines belonging to other pharmacological groups. For pharmacological purposes, the division into psycholeptic, psychoanaleptic, and psychodysleptic drugs (hallucinogenic and psychotomimetic) is introduced. For practical purposes, people speak more often about neuroleptics, antidepressants, and anxiolytics. The pharmacological classification is also applied to the chemical classification taking into account the structure of the

Piotr Kawczak

p99p@gumed.edu.pl

1 Department of Pharmaceutical Chemistry, Faculty of Pharmacy with Subfaculty of Laboratory Medicine, Medical University of Gdańsk, Gdańsk 80-416, Poland

2 POLPHARMA SA Pharmaceutical Works, Starogard, Gdański 83-200, Poland

3 Institute of Health Sciences, Division of Human Anatomy and Physiology, Pomeranian University of Słupsk, Słupsk 76-200, Poland molecules of the considered drugs (Hendriksen and Groenink 2015; Stahl 2013; Dean 2011; Spiegel 2003).

Psychotropic agents have often been the subject of many works in the field of structure-properties, such as the relationship between the enthalpy of complexation of charge-transfer (CT) complexes with chloranil and their biological activity by a series of neuroleptics (Saucin and van der Vorst 1972), relationships between lipophilicity indices determined by RP-HPLC methods (as well as constants dissociation) and biological activity (Unger and Chiang 1981), pharmacological classification based on retention data in HPLC systems (Nasal et al. 1997) or attempts to predict biological activity for tricyclic neuroleptics and antidepressants based on quantum-chemical calculations of energy values of boundary orbitals e.g. HOMO and LUMO (Cogordan et al. 1999).

The subject of this work was the analysis for data from ab initio calculations and the biological activity data as well as physicochemical parameters presented in the cited papers (Saucin and van der Vorst 1972; Unger and Chiang 1981; Davis and Brody 1966). In addition, chromatographic retention data (Nasal et al. 1997) were also available for some of the compounds considered, which were used as variables dependent on structural parameters.

The aim of this research was to demonstrate both common and differentiating features of the analyzed chemical 
compounds both in physicochemical and pharmacological terms and confirmed the chemometrics relationship between nonempirical parameters characterizing chemical structure and biological activity.

\section{Materials and methods}

\section{Molecules}

The following compounds (Fig. 1), considered in the cited papers (Unger and Chiang 1981; Saucin and van der Vorst 1972; Davis and Brody 1966) were selected for the study: acoperon, benperidol, droperidol, haloperidol, spiroperidol, and triperidol from the group of butyrophenone neuroleptics, acetophenazine, chlorpromazine, chlorprothixen, dixyrazine, fluphenazine, levomepromazine, perphenazine, prochlorperazine, promazine, promethazine, thioproperazine, thioridazine, trifluoperazine, and trifluopromazine from the group of tricyclic neuroleptics; and: amitriptiline, clomipramine, desipramine, imipramine, and nortriptiline from the group of tricyclic antidepressants. Data on their structure were available for these compounds.

\section{Biological activity and physicochemical parameters data}

The study includes literature data on biological activity: D dose $(\mathrm{mg} / \mathrm{kg}) 50 \%$ reduction in motor activity of rats expressed as $\log (1 / \mathrm{D})$ for neuroleptics of butyrophenone derivatives and parts of tricyclic neuroleptics (Saucin and van der Vorst 1972); histamine release as $\log \left(1 / \mathrm{ED}_{50} \times 10\right.$ ${ }^{-3}$ ) for parts of tricyclic neuroleptics and tricyclic antidepressants (Unger and Chiang 1981); ATPase activity inhibition as $\log [\% /(100-\%)]$ at $1 \times 10^{-4} \mathrm{M}$ for parts of tricyclic neuroleptics and imipramine (Davis and Brody 1966). The enthalpy values of $-\Delta H(\mathrm{kcal} / \mathrm{mol})$ formation of neuroleptic CTs with chloranil (Saucin and van der Vorst 1972) obtained on the basis of spectroscopic measurements of CT complexes, and concern: neuroleptics of the butyrophenone and chlorpromazine derivatives, chlorprothixen, levomepromazine, prochlorperazine, and thioproperazines from the group of tricyclic neuroleptics.

\section{Chromatographic retention data}

Chromatographic data comes from the work of Nasal et al. (1997) and relate to the compounds: chlorpromazine, chlorprothixene, fluphenazine, perphenazine, prochlorperazine, promazine, promethazine, thioridazine, trifluoperazine, trifluopromazine from the group of tricyclic neuroleptics, and: clomipramine, desipramine and imipramine from the group of tricyclic antidepressants. These are logarithm values of the retention coefficients determined on Chiral AGP $\left(\log k_{\mathrm{AGP}}\right)$ fillings and artificial IAM.PC.MG membranes $\left(\log k_{\text {IAM }}\right)$ but also logarithm values of hydrophobicity coefficients determined by the polycratic method on suplex pKb-100 fillings pH 2.5 and $7.4\left(\log k_{\mathrm{w} 2.5 \mathrm{Su}}, \log \right.$ $\left.k_{\mathrm{w} 7.4 \mathrm{Su}}\right)$, Spheri RP-18 pH 2.5 and $7.0\left(\log k_{\mathrm{w} 2.5 \mathrm{Sp}}\right.$, $\log$ $\left.k_{\mathrm{w} 7.05 \mathrm{p}}\right)$, Aluspher RP select B pH $7.3\left(\log k_{\mathrm{w} 7.3 \mathrm{Al}}\right)$ and Unisphere-PBD pH $11.7\left(\log k_{\mathrm{w} 11.7 \mathrm{Un}}\right)$.

\section{Molecular descriptors}

Nonempirical structural indicators-quantum-chemical indicators were calculated during the study. The structure of the tested compounds was studied by molecular modeling using the Gaussian 03W program (Gaussian Inc., Wallingford, CT, USA). The geometry of the molecules was optimized by the Hartree-Fock 6-31G (d, p) method (other designation is 6-31G**) (http://www.gaussian.com/). Among the quantum-chemical indicators were considered: total energy (TE), electronic spatial range (ESE) - the spatial extent of the molecule is defined as the surface covering the volume around the molecule beyond which the electron density is $<0.001 \mathrm{eBohr}^{-3}$, and describes the sensitivity of the molecule to the electric field), the energy of the highest occupied molecular orbitals (E_HOMO), the energy of the lowest unoccupied molecular orbitals (E_LUMO) and the energy difference HOMO and LUMO determined as energy gap (EG). In addition, the following values were used: the largest positive electron charge on atoms (MAX_POS), the largest negative electron charge on atoms (MAX_NEG), the difference between the highest positive and negative charge $(\Delta Q)$, total dipole moment (TDM), and isotropic polarization (IPOL). The values of total energy were expressed in atomic energy units a.u. or hartree ( 1 hartree $=2625.552$ $\mathrm{kJ} \cdot \mathrm{mol}^{-1}$ or $627.5095 \mathrm{kcal} \cdot \mathrm{mol}-1$ or $\left.27.2116 \mathrm{eV}\right), \mathrm{HOMO}$ energies, LUMO, and energy break were expressed in $\mathrm{eV}$ (the above values were converted from a.u. to $\mathrm{eV}$ ), electronic spatial range in $\mathrm{eBohr}^{-3}$. The values of electron density and electron charges on atoms are in units of elementary charge $\left(\mathrm{e}^{-}\right)$, dipole moment is expressed in debay (D) and isotropic polarizability in $\mathrm{Bohr}^{3}(\mathrm{Bohr}=0.5292 \cdot 10$ ${ }^{-10} \mathrm{~m}=0.5292 \AA$ ). Finally, for the whole group of molecules Dragon 7.0 (Kode Chemoinformatics, Pisa, Italy) software was used to calculate huge set -5270 of extra descriptors (Todeschini and Consonni 2010; Dragon 7 molecular descriptors $2018 \mathrm{https} / / / \mathrm{chm}$.kode-solutions.net/ products_dragon.php).

\section{Statistical analysis}

The data examined the biological activity, physicochemical and retention parameters of compounds were related to their structural indicators using multiparametric regression 
Table 1 The numerical values of 10 structural parameters derived from Gaussian quantum-chemical calculations for all 25 compounds studied

\begin{tabular}{|c|c|c|c|c|c|c|c|c|c|c|}
\hline Compound & TE & ESE & E_HOMO & E_LUMO & EG & MAX_POS & MAX_NEG & $\Delta Q$ & TDM & IPOL \\
\hline Aceperon & -1283.84 & 21234.30 & -8.9739 & 2.5415 & 11.5154 & 0.7254 & -0.7283 & 1.4537 & 6.3837 & 242.90 \\
\hline Acetophenazine & -1598.45 & 14981.03 & -7.6523 & 2.3717 & 10.0240 & 0.5402 & -0.7950 & 1.3352 & 1.7719 & 259.79 \\
\hline Amitriptiline & -825.22 & 6515.35 & -8.1413 & 3.5380 & 11.6793 & 0.1801 & -0.5770 & 0.7571 & 1.1089 & 199.06 \\
\hline Benperidol & -1259.67 & 20740.83 & -7.9418 & 2.6286 & 10.5704 & 1.0460 & -0.8566 & 1.9026 & 3.1622 & 228.43 \\
\hline Chlorpromazine & -1620.72 & 8397.08 & -7.7290 & 3.2460 & 10.9750 & 0.3143 & -0.7846 & 1.0989 & 2.7007 & 201.34 \\
\hline Chlorprothixene & -1603.56 & 8999.30 & -7.9483 & 2.6438 & 10.5921 & 0.2668 & -0.5915 & 0.8583 & 2.2333 & 211.05 \\
\hline Clomipramine & -1301.28 & 8666.11 & -8.0945 & 3.5056 & 11.6001 & 0.2790 & -0.7897 & 1.0687 & 2.7070 & 204.08 \\
\hline Desipramine & -803.36 & 6526.89 & -7.8879 & 3.7848 & 11.6727 & 0.2804 & -0.7888 & 1.0692 & 0.9264 & 183.15 \\
\hline Dixyrazine & -1638.63 & 18634.72 & -7.5078 & 3.5674 & 11.0752 & 0.3471 & -0.8013 & 1.1484 & 1.4385 & 269.39 \\
\hline Droperidol & -1258.48 & 19155.09 & -7.9960 & 2.5135 & 10.5095 & 1.0333 & -0.8941 & 1.9274 & 5.3046 & 225.82 \\
\hline Fluphenazine & -1782.30 & 16638.00 & -7.7804 & 2.9826 & 10.7630 & 1.1718 & -0.7955 & 1.9673 & 4.7537 & 246.12 \\
\hline Haloperidol & -1571.75 & 17976.49 & -8.7881 & 2.4400 & 11.2281 & 0.5509 & -0.6697 & 1.2207 & 3.9820 & 220.16 \\
\hline Imipramine & -842.38 & 7331.05 & -7.8795 & 3.7897 & 11.6692 & 0.2800 & -0.7892 & 1.0693 & 0.7071 & 193.13 \\
\hline Levomepromazine & -1314.75 & 8340.75 & -7.3870 & 3.6136 & 11.0006 & 0.4185 & -0.8025 & 1.2209 & 1.5158 & 215.72 \\
\hline Nortriptiline & -786.19 & 6148.49 & -8.0906 & 3.5102 & 11.6008 & 0.2710 & -0.6326 & 0.9036 & 1.6374 & 189.70 \\
\hline Perphenazine & -1905.57 & 14014.45 & -7.6751 & 3.2868 & 10.9619 & 0.3607 & -0.7944 & 1.1551 & 3.8012 & 246.35 \\
\hline Prochlorperazine & -1791.68 & 11122.23 & -7.6588 & 3.2955 & 10.9543 & 0.3613 & -0.7944 & 1.1557 & 2.0514 & 233.31 \\
\hline Promazine & -1161.82 & 7343.41 & -7.1788 & 3.5344 & 10.7132 & 0.3786 & -0.8293 & 1.2078 & 2.5886 & 193.37 \\
\hline Promethazine & -1161.82 & 5419.31 & -7.2528 & 3.6487 & 10.9015 & 0.3699 & -0.8095 & 1.1793 & 2.1065 & 187.65 \\
\hline Spiroperidol & -1298.66 & 20148.70 & -8.6112 & 2.6278 & 11.2390 & 0.7960 & -0.7296 & 1.5256 & 4.3300 & 234.58 \\
\hline Thioproperazine & -2013.05 & 14948.37 & -7.8264 & 2.6357 & 10.4621 & 1.7000 & -0.7947 & 2.4947 & 5.9427 & 270.47 \\
\hline Thioridazine & -1714.32 & 10820.72 & -7.6270 & 3.0993 & 10.7263 & 0.3470 & -0.7869 & 1.1339 & 2.6694 & 247.11 \\
\hline Trifluoperazine & -1668.40 & 12802.53 & -7.7701 & 2.9804 & 10.7505 & 1.1715 & -0.7956 & 1.9672 & 2.5362 & 232.44 \\
\hline Trifluopromazine & -1497.44 & 10070.78 & -7.8596 & 2.9567 & 10.8163 & 1.1740 & -0.7842 & 1.9583 & 3.3624 & 200.41 \\
\hline Triperidol & -1448.48 & 21739.60 & -8.8887 & 2.4065 & 11.2952 & 1.1740 & -0.6691 & 1.8432 & 4.7615 & 219.02 \\
\hline
\end{tabular}

analysis/multiple regression analysis (MLR) with a stepwise progressive method together with principal component analysis (PCA) and factor analysis (FA) implemented in Statistica 13 (StatSoft, Tulsa, OK, USA) on a personal computer. In PCA, matrix of correlations has been diagonalized and in FA Varimax rotation has been performed.

\section{Results and discussion}

The numerical values of all the 10 structural parameters derived from quantum-chemical calculations for all 25 compounds examined are shown in Table 1, the chromatographic retention parameters in Table 2 and finally the biological activity values and physicochemical parameters for selected compounds in Table 3.

First of all, the analysis of PCA was performed for the geometry-optimized structures. At the outset, a PCA analysis was carried out of only non-empirical data in order to check whether and to what extent they could be useful in order to classify the compounds in question. The following results were obtained: $\mathrm{PC} 1$ is about $50.2 \%, \mathrm{PC} 2$ is equal to
22.6\% and PC3 10.9\%. PC1 has the greatest impact on the maximum positive charge on atoms (MAX_POS), the difference between the highest positive and negative charge on atoms $(\Delta Q)$ and isotropic polarization (IPOL). It is interesting that electrons are considered polar parameters, while bulk parameters (TE, ESE) have an approximately smaller impact on PC1. On the other hand, PC2 influences mostly the largest negative electron charge on atoms (MAX_NEG) and the energy of the highest occupied molecular orbital (E_HOMO). PC3 has the greatest impact on IPOL and again $\Delta Q$ but also appears MAX_NEG and MAX_POS with smaller impact Fig. 1.

Factor analysis (FA) performed with the same calculation conditions coincides completely with the results for the molecules obtained by PCA method (FA1 $\sim 50.2 \%$ and FA2 22.6\%).

Figure 2 represents scatter plots of the scores according to performed PCA and FA. Analyzing PCA 3D scatter plots of the score we can distinguish three type of clusters; the first one includes: acepron, benperidol, droperidol, haloperidol, spiroperidol, triperidol, and also fluphenazine, trifluopromazine, trifluoperazine, thioproperazine; the second 
Table 2 Chromatographic parameter values of selected antipsychoctic drugs

\begin{tabular}{lllllllll}
\hline Compound & $\log \mathrm{k}_{\mathrm{AGP}}$ & $\log \mathrm{k}_{\mathrm{IAM}}$ & $\log \mathrm{k}_{\mathrm{w} 2.5 \mathrm{Su}}$ & $\log \mathrm{k}_{\mathrm{w} 7.4 \mathrm{Su}}$ & $\log \mathrm{k}_{\mathrm{w} 2.5 \mathrm{Sp}}$ & $\log \mathrm{k}_{\mathrm{w} 7.0 \mathrm{Sp}}$ & $\log \mathrm{k}_{\mathrm{w} 7.3 \mathrm{Al}}$ & $\log \mathrm{k}_{\mathrm{w} 11.7 \mathrm{Un}}$ \\
\hline Chlorpromazine & 2.131 & 1.435 & 1.595 & 4.051 & 1.935 & 2.632 & 3.309 & 4.076 \\
Chlorprothixene & 2.206 & 1.533 & 1.597 & 4.642 & 2.244 & 2.417 & 4.440 & 4.235 \\
Clomipramine & 2.005 & 1.391 & 0.781 & 4.144 & 2.353 & 2.473 & 4.115 & 3.910 \\
Desipramine & 1.595 & 1.031 & 2.134 & 3.020 & 2.015 & 2.341 & 3.171 & 2.888 \\
Fluphenazine & 2.159 & 1.496 & 1.683 & 4.554 & 2.922 & 2.688 & 4.067 & 3.352 \\
Imipramine & 1.670 & 1.097 & 1.391 & 3.535 & 2.082 & 3.158 & 3.133 \\
Perphenazine & 2.283 & 1.393 & 1.635 & 4.305 & 2.997 & 3.092 & 3.256 & 3.020 \\
Prochlorperazine & 2.614 & 1.726 & 1.452 & 4.878 & 1.843 & 2.421 & 4.395 \\
Promazine & 1.890 & 1.165 & 1.556 & 3.492 & 2.338 & 2.808 & 3.794 & 3.523 \\
Promethazine & 1.833 & 1.508 & 1.693 & 4.081 & 1.132 & 3.169 & 3.069 \\
Thioridazine & 2.448 & 1.752 & 2.113 & 4.260 & 2.055 & 2.924 & 3.182 \\
Trifluoperazine & 2.388 & 1.820 & 1.778 & 4.948 & 1.792 & 2.644 & 5.022 & 3.216 \\
Triflupromazine & 1.976 & 1.514 & 1.960 & 4.409 & 2.533 & 2.638 & 3.790
\end{tabular}

Table 3 Biological activity/physicochemical properties values of selected antipsychotic drugs

\begin{tabular}{lllll}
\hline Compound & $-\Delta H$ & $\log 1 / \mathrm{D}$ & $\begin{array}{l}\log (1 / \\
\left.\mathrm{ED}_{50} \times 10^{-3}\right)\end{array}$ & $\begin{array}{l}\text { log ATPase } \\
\text { activity }\end{array}$ \\
\hline Aceperon & 0.37 & -1.623 & - & - \\
Acetophenazine & - & - & 2.12 & - \\
Amitriptiline & - & - & 1.85 & - \\
Benperidol & 1.82 & 1.523 & - & - \\
Chlorpromazine & 5.12 & -0.301 & 2.52 & -0.21 \\
Chlorprothixene & 0.19 & 0.022 & 2.57 & - \\
Clomipramine & - & - & 2.22 & - \\
Desipramine & - & - & 1.58 & - \\
Dixyrazine & 4.78 & -0.398 & - & - \\
Droperidol & 0.96 & 1.284 & - & - \\
Fluphenazine & 0.00 & 1.097 & - & - \\
Haloperidol & 5.16 & 1.284 & - & - \\
Imipramine & - & - & 1.79 & -0.99 \\
Levomepromazine & 3.42 & -0.330 & - & - \\
Nortriptiline & - & - & 1.76 & - \\
Perphenazine & - & - & 2.63 & 0.09 \\
Prochlorperazine & 0.00 & 0.137 & 2.75 & 0.87 \\
Promazine & - & - & 2.05 & -1.17 \\
Promethazine & - & - & 1.82 & -0.37 \\
Spiroperidol & 0.69 & 2.000 & - & - \\
Thioproperazine & 0.00 & 0.602 & - & - \\
Thioridazine & - & - & 2.72 & 0.79 \\
Trifluoperazine & - & - & 2.92 & 1.69 \\
Trifluopromazine & - & - & - & 0.27 \\
Triperidol & 5.78 & 1.456 & - & - \\
\hline & & & & \\
\hline
\end{tabular}

cluster containes: amitriptiline, clomipramine, desipramine, imipramine, nortriptiline also chlorpromazine, levomepromazine, promazine, promethazine; the third cluster includes: acetophenazine, chlorprothixene, dixyrazine, perphenazine, prochlorperazine, and thioridazine. We can observe that the first cluster mostly involves neuroleptics derived from butyrophenone and other very potent neuroleptics, the second one mostly belongs to tricyclic antidepressants (dibenzoazepine and dibenzoheptadiene derivatives), and the third one mostly to tricyclic neuroleptics.

On the other hand, analyzing FA 2D scatter plots of scores we can also distinguish three types of clusters: the first one includes: acepron, droperidol, haloperidol, and spiroperidol; the second: amitriptiline, clomipramine, desipramine, imipramine nortriptiline, and chlorprothixene and finally the third one includes all the others studied psychoactive drugs.

Statistically significant Gaussian parameters characterizing biological activity are presented in the following equations:

$$
\begin{aligned}
& \log \left(1 / \mathrm{ED}_{50} \times 10^{-3}\right)=k_{0}-0.9150 T E( \pm 0.1165) \\
& \quad R=0.9150, R^{2}=0,8372, F=61.7126, s=0.1813, p< \\
& 0.0001, n=14, k_{0}=0 \\
& \log \text { ATPase activity }=k_{0}-0.7896 \text { E_LUMO }( \pm 0.2319)
\end{aligned}
$$

$$
\begin{aligned}
& R=0.7896, R^{2}=0.6235, F=11.5949, s=0.6559, p= \\
& 0.0114, n=9, k_{0}=0
\end{aligned}
$$

Similarly, statistically significant Gaussian parameters characterizing chromatographic retention values are presented below:

$\log \mathrm{k}_{\mathrm{AGP}}=k_{0}-1.2633 \mathrm{TE}( \pm 0.1299)-0.4892 \mathrm{TDM}( \pm 0.1299)$

$R=0.9608, R^{2}=0.9231, F=60.0945, s=0.3036, p<$ $0.0001, n=13, k_{0}=0$

$\log \mathrm{k}_{\mathrm{IAM}}=k_{0}-0.7713 \mathrm{TE}( \pm 0.1919)$ 
Figure 1 Structural formulas of compounds studied
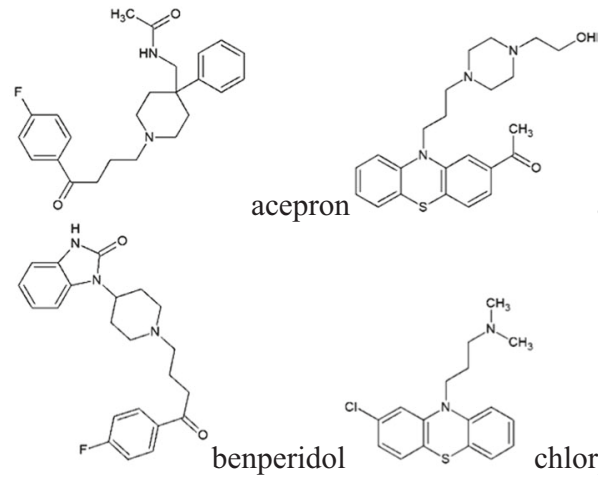

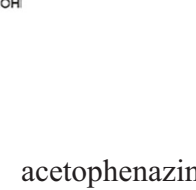

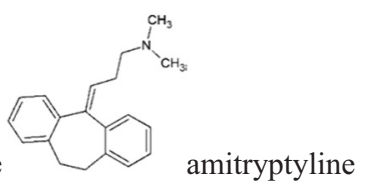

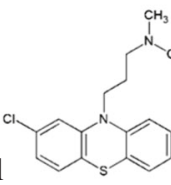

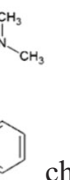
chlorpromazine

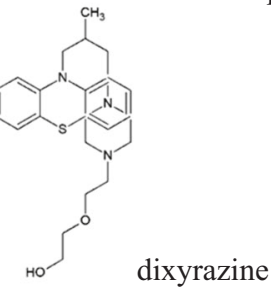<smiles>CC(C)CCCN1c2ccccc2Cc2ccc(Cl)cc21</smiles><smiles>CCCCCN1c2ccccc2CCc2ccccc21</smiles>

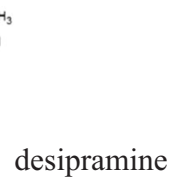

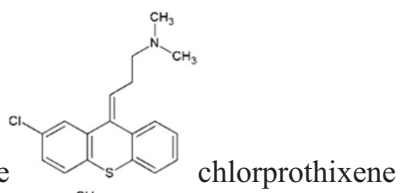<smiles>O=C(CCCN1CC=C(n2c(=O)[nH]c3ccccc32)CC1)c1ccc(F)cc1</smiles><smiles>OCCC1CCC(CCCN2Cc3ccccc3Sc3ccc(C(F)(F)F)cc32)CC1</smiles>

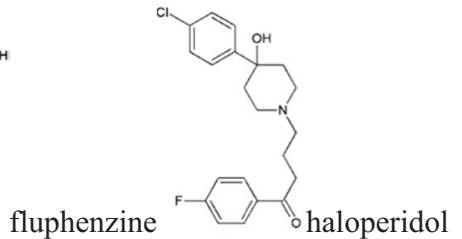<smiles>CC(C)CCCN1c2ccccc2CCc2ccccc21</smiles>

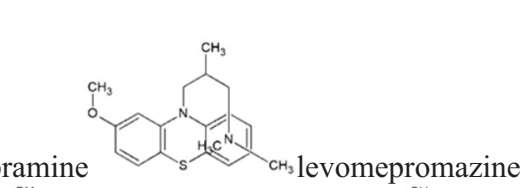

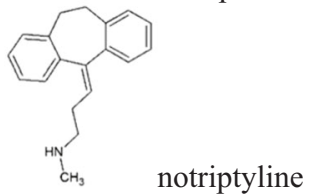<smiles>OCCN1CCC(N2CCCCC23Nc2ccccc2Nc2ccc(Cl)cc23)CC1</smiles>

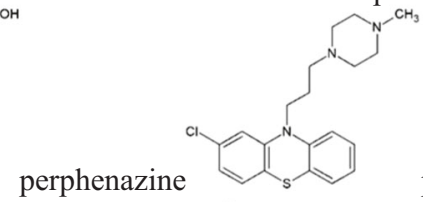
azine
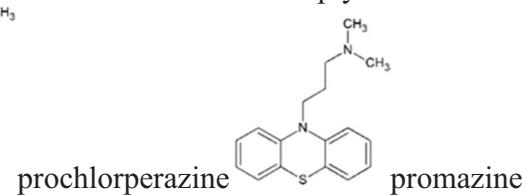<smiles>CC1Cc2c3ccc4c5ccccc5sc5ccccc5n(c24)[C@@H](C[C@H](C)[C@H]3C)c2ccccc21</smiles>
spiroperidol<smiles>CN(C)C(=O)c1ccc2c(c1)N(CCCC1CCC(N)CC1)c1ccccc1S2</smiles>

thioproperazine<smiles>COc1ccc2c3cccnc3sc3ccccc3n(CCC3CCCCN3C)c2c1</smiles>

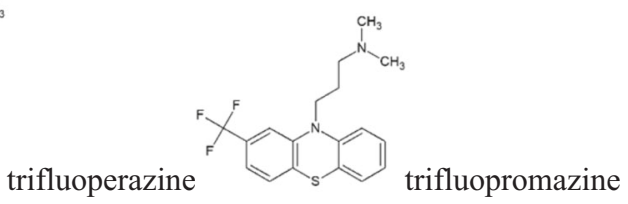<smiles>O=C(C=CCCCN1CCC(c2cccc(F)c2)CC1)c1ccc(F)cc1</smiles> 

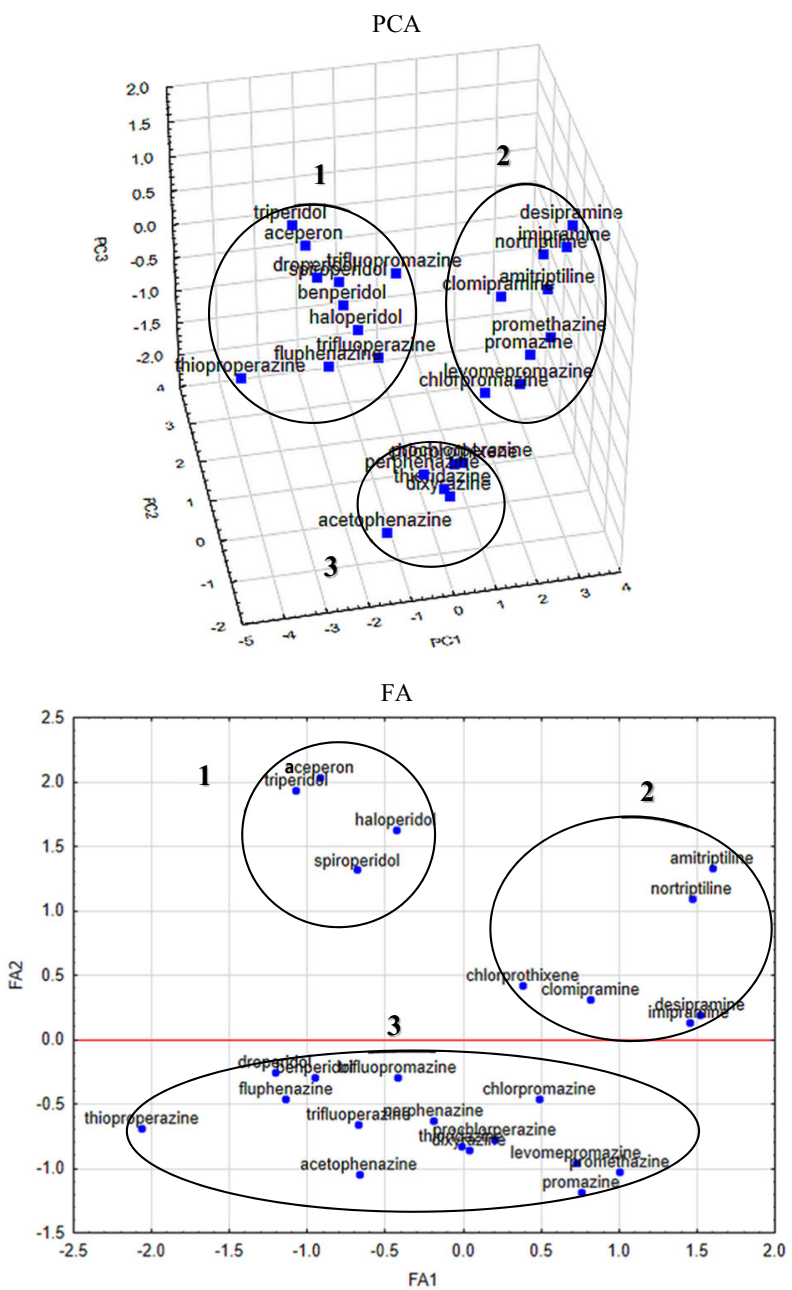

Figure 2 Three-dimensional scatter plots of the scores derived from the quantum-chemical calculations of structural parameters of the first three components obtained using PCA (PC1 50.21\%, PC2 22.63\%, PC3 10.89\%) together with two-dimensional plot of scores the first two factors obtained by FA (FA1 50.21\%, FA2 22.63\%)

$$
\begin{aligned}
& R=0.7713, R^{2}=0.5949, F=16.1514, s=0.6648, p= \\
& 0.0020, n=13, k_{0}=0 \\
& \log \mathrm{k}_{\mathrm{w} 7.4 \mathrm{Su}}=k_{0}-0.8364 \mathrm{TE}( \pm 0.1652)
\end{aligned}
$$$$
R=0.8364, R^{2}=0.6996, F=25.6215, s=0.5724, p=
$$$$
0.0004, n=13, k_{0}=0
$$$$
\log \mathrm{k}_{\mathrm{w}} 2.5 \mathrm{Sp}=k_{0}+0.6378 \mathrm{ESE}( \pm 0.2322)
$$$$
R=0.6378, R^{2}=0.4068, F=7.5449, s=0.8044, p=
$$$$
0.0190, n=13, k_{0}=0
$$$$
\log \mathrm{k}_{\mathrm{w} 7.3 \mathrm{Al}}=k_{0}-0.5936 E L U M O( \pm 0.2426)
$$$$
R=0.5936, R^{2}=0.3524, F=5.9859, s=0.8405, p=
$$$$
0.0324, n=13, k_{0}=0
$$$$
\log \mathrm{k}_{\mathrm{w} 11.7 \mathrm{Un}}=k_{0}-0.6671 \mathrm{E} \_\mathrm{LUMO}( \pm 0.2246)
$$

$$
R=0.6671, R^{2}=0.4450, F=8.8226, s=0.0127, p=
$$
$0.7780, n=13, k_{0}=0$
Obtained results in most with TE and E_LUMO (TDM and ESE in individual cases) may indicate the specific nature of interactions between the drug molecule and the receptor.

In the final step, progressive stepwise multiple regression analysis was performed but with the use of a huge set of additional descriptors obtained by the professional software for the analyzed compounds with owned biological activity and chromatographic retention parameter values. Model equations for statistically significant descriptors are presented on below set of derived equations.

Biological activity/physicochemical properties values described by the Dragon software are as follows:

$$
\begin{gathered}
-\Delta \mathrm{H}[\mathrm{kcal} / \mathrm{mol}]=k_{0}+0.5040 \mathrm{CATS} 2 \mathrm{D} \_09 \_\mathrm{DA} \\
( \pm 0.1326)-1.0654 \mathrm{RDF} 095 \mathrm{v}( \pm 0.2195)+ \\
0.6750 \mathrm{H} 4 \mathrm{~m}( \pm 0.2357)
\end{gathered}
$$

$R=0.9537, R^{2}=0.9095, F=30.1882, s=0.3472, p<$ $0.0001, n=13, k_{0}=0$

$$
\begin{aligned}
& \log 1 / \mathrm{D}=k_{0}+0.5926 \mathrm{H}-053( \pm 0.0630)+0.4112 \mathrm{PJI} 3 \\
&( \pm 0.0614)+0.3169 \mathrm{AVS} \_\mathrm{B}(\mathrm{e})( \pm 0.0584)
\end{aligned}
$$

$$
R=0.9858, R^{2}=0.9718, F=103.6188, s=0.1937, p<
$$
$0.0001, n=13, k_{0}=0$

$$
\begin{aligned}
& \log \left(1 / \mathrm{ED}_{50} \times 10^{-3}\right)=k_{0}+0.8553 \mathrm{DISPm}( \pm 0.0647)+ \\
& 0.3122 \operatorname{Mor} 08 \mathrm{u}( \pm 0.0585)+0.1797 \operatorname{MATS} 6 \mathrm{~s}( \pm 0.0566)
\end{aligned}
$$

$$
R=0.9873, R^{2}=0.9748, F=128.4855, s=0.1813, p<
$$
$0.0001, n=14, k_{0}=0$

$\log$ ATPase activity $=k_{0}+0.7842$ Eig04_AEA

$$
\text { (bo) }( \pm 0.0747)-0.2958 \text { GATS5s }( \pm 0.0747)
$$

$$
R=0.9891, R^{2}=0.9783, F=135.5797, s=0.1699, p<
$$
$0.0001, n=9, k_{0}=0$

Analogous chromatographic retention parameters described by the Dragon software are presented below:

$$
\begin{gathered}
\log \mathrm{k}_{\mathrm{AGP}}=k_{0}+1.2632 \mathrm{H} 1 \mathrm{v}( \pm 0.1175)- \\
0.2847 \mathrm{DISPi}( \pm 0.0994)-0.1993 H 2 u( \pm 0.0859)
\end{gathered}
$$

$$
R=0.9804, R^{2}=0.9612, F=74.4953, s=0.2272, p<
$$
$0.0001, n=13, k_{0}=0$

$$
\begin{aligned}
\log \mathrm{k}_{\mathrm{IAM}} & =k_{0}-0.8764 \mathrm{X} 1 \mathrm{~A}( \pm 0.0826)-0.4126 \mathrm{GATS} 5 \mathrm{~m} \\
& ( \pm 0.0697)-0.2502 \operatorname{Mor} 06 \mathrm{p}( \pm 0.0817)
\end{aligned}
$$


Table 4 List of statistically significant molecular descriptors characterizing biological activity/physicochemical properties/chromatographic retention obtained from Dragon software (Eqs. 9-18)

\begin{tabular}{|c|c|c|}
\hline Descriptor & Full name & Block \\
\hline \multicolumn{3}{|c|}{ Biological activity/physicochemical properties } \\
\hline CATS2D_09_DA & $\begin{array}{l}\text { CATS (Chemically Advanced Template Search) 2D Donor-Acceptor } \\
\text { at lag } 09\end{array}$ & CATS2D \\
\hline RDF095v & $\begin{array}{l}\text { Radial Distribution Function - } 095 \text { / weighted by van der Waals } \\
\text { volume }\end{array}$ & RDF descriptors \\
\hline $\mathrm{H} 4 \mathrm{~m}$ & $\mathrm{H}$ autocorrelation of lag 4/weighted by mass & $\begin{array}{l}\text { GETAWAY (GEometry, Topology, and Atom-Weights AssemblY) } \\
\text { descriptors }\end{array}$ \\
\hline H-053 & $\mathrm{H}$ attached to $\mathrm{C} 0(\mathrm{sp} 3)$ with $2 \mathrm{X}$ attached to next $\mathrm{C}$ & Atom-centered fragments \\
\hline PJI3 & 3D Petitjean shape index & Geometrical descriptors \\
\hline AVS_B(e) & $\begin{array}{l}\text { Average vertex sum from Burden matrix weighted by Sanderson } \\
\text { electronegativity }\end{array}$ & 2D matrix-based descriptors \\
\hline DISPm & Displacement value/weighted by mass & Geometrical descriptors \\
\hline Mor08u & Signal 08/unweighted & $\begin{array}{l}\text { 3D-MoRSE (3D-Molecule Representation of Structures based on Electron } \\
\text { diffraction) descriptors }\end{array}$ \\
\hline MATS6s & Moran autocorrelation of lag 6 weighted by I-state & 2D autocorrelations \\
\hline Eig04_AEA(bo) & $\begin{array}{l}\text { eigenvalue } \mathrm{n} .4 \text { from augmented edge adjacency mat. weighted by } \\
\text { bond order }\end{array}$ & Edge adjacency indices \\
\hline GATS5s & Geary autocorrelation of lag 5 weighted by I-state & 2D autocorrelations \\
\hline \multicolumn{3}{|l|}{ Retention parameters } \\
\hline $\mathrm{H} 1 \mathrm{v}$ & $\mathrm{H}$ autocorrelation of lag 1 /weighted by van der Waals volume & GETAWAY descriptors \\
\hline DISPi & Displacement value/weighted by ionization potential & Geometrical descriptors \\
\hline $\mathrm{H} 2 \mathrm{u}$ & $\mathrm{H}$ autocorrelation of lag 2/unweighted & GETAWAY descriptors \\
\hline $\mathrm{X} 1 \mathrm{~A}$ & Average connectivity index of order 1 & Connectivity indices \\
\hline GATS5m & Geary autocorrelation of lag 5 weighted by mass & 2D autocorrelations \\
\hline Mor06p & Signal $06 /$ weighted by polarizability & 3D-MoRSE descriptors \\
\hline $\mathrm{R} 2 \mathrm{v}$ & $\mathrm{R}$ autocorrelation of lag $2 /$ weighted by van der Waals volume & GETAWAY descriptors \\
\hline CMC-50 & Ghose-Viswanadhan-Wendoloski CMC drug-like index at $50 \%$ & Drug-like indices \\
\hline $\mathrm{R} 1 \mathrm{e}+$ & $\begin{array}{l}\mathrm{R} \text { maximal autocorrelation of lag } 1 / \text { weighted by Sanderson } \\
\text { electronegativity }\end{array}$ & GETAWAY descriptors \\
\hline Mor09i & Signal $09 /$ weighted by ionization potentia & 3D-MoRSE descriptors \\
\hline $\mathrm{B} 08[\mathrm{C}-\mathrm{N}]$ & Presence/absence of $\mathrm{C}-\mathrm{N}$ at topological distance 8 & 2D Atom Pairs \\
\hline $\mathrm{H} 6 \mathrm{~m}$ & $\mathrm{H}$ autocorrelation of lag $6 /$ weighted by mass & GETAWAY descriptors \\
\hline ATS6s & $\begin{array}{l}\text { Broto-Moreau autocorrelation of lag } 6 \text { (log function) weighted by I- } \\
\text { state }\end{array}$ & 2D autocorrelations \\
\hline $\mathrm{H} 4 \mathrm{i}$ & $\mathrm{H}$ autocorrelation of lag 4/weighted by ionization potential & GETAWAY descriptors \\
\hline ALOGP2 & Squared Ghose-Crippen octanol-water partition coeff. $\left(\log \mathrm{P}^{\wedge} 2\right)$ & Molecular properties \\
\hline HATS1v & $\begin{array}{l}\text { Leverage-weighted autocorrelation of lag } 1 / \text { weighted by van der } \\
\text { Waals volume }\end{array}$ & GETAWAY descriptors \\
\hline $\mathrm{R} 8 \mathrm{v}$ & $\mathrm{R}$ autocorrelation of lag 8 /weighted by van der Waals volume & GETAWAY descriptors \\
\hline
\end{tabular}

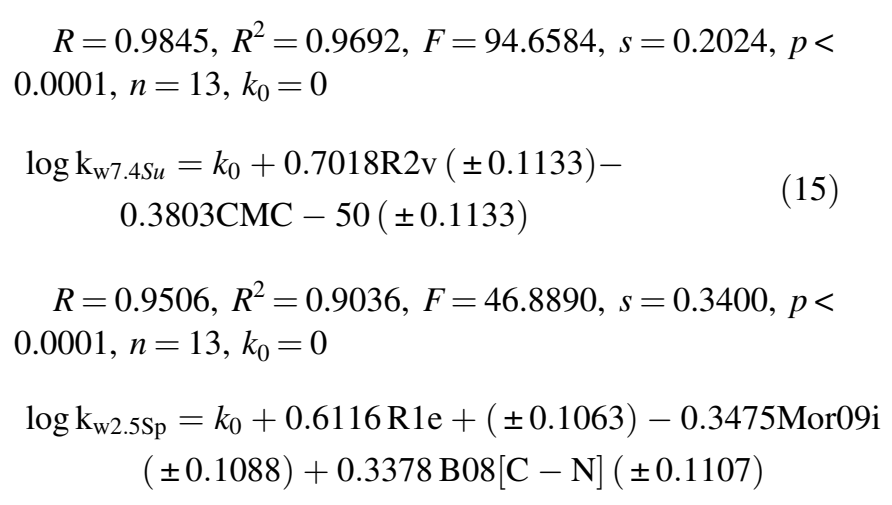

$$
\begin{gathered}
R=0.9532, R^{2}=0.9086, F=29.8380, s=0.3490, p< \\
0.0001, n=13, k_{0}=0 \\
\log \mathrm{k}_{\mathrm{w} 7.3 \mathrm{Al}}=k_{0}+1.2948 \mathrm{H} 6 \mathrm{~m}( \pm 0.1279)-1.4089 \text { ATS6s } \\
( \pm 0.1882)+0.8301 \mathrm{H} 4 \mathrm{i}( \pm 0.1638)
\end{gathered}
$$

$R=0.9718, R^{2}=0.9444, F=50.8709, s=0.2725, p<$ $0.0001, n=13, k_{0}=0$

$$
\log \mathrm{k}_{\mathrm{w} 11.7 \mathrm{Un}}=k_{0}+0.4900 \mathrm{ALOGP} 2( \pm 0.1072)+
$$$$
0.3580 \mathrm{HATS} 1( \pm 0.0598)+0.3380 \mathrm{R} 8 \mathrm{v}( \pm 0.1004)
$$ 
$R=0.9873, R^{2}=0.9748, F=115.9598, s=0.1834, p<$ $0.0001, n=13, k_{0}=0$

The full list of molecular descriptors obtained from Dragon software with their designations is presented in Table 4.

Performed predictions with the use of professional software and the wide range of molecular descriptors provide more detail information about the studied molecules.

The obtained statistically significant molecular descriptors belong to different classes but we can distinguish some of the common one's classes between them. In our opinion it is difficult to indicate the most important equation between the proposed because the datasets are relatively small, therefore we wanted to focus rather on the same block of descriptors appearing in the presented equations.

In the case of biological activity/physicochemical properties of analyzed psychoactive drugs, the most often appeared geometrical descriptors together with GETAWAY and atom fragments descriptors followed by the 2D autocorrelations and 3D-MoRSE descriptors. Experimental equations confirmed the very important role of geometric and topologic properties of the molecules together with electronic ones. Furthermore, interesting information we received also analyzing the retention parameters values on different chromatographic columns and in different chromatographic conditions. There was a confirmation in eight of the experimental cases particularly important role of GETAWAY descriptors and also molecular properties descriptors (e.g. ALOGP2, characterizing octanol-water partition coefficient) followed by appeared again 2D autocorrelations and 3D-MoRSE descriptors, so we can observe that similar group of descriptors play a dominant role in pharmacological role and physicochemical properties of examined psychoactive structures.

\section{Conclusion}

Based on the above discussion, the results can be drawn as follows. The largest influence on the values of both biological activity/physicochemical properties and chromatographic retention parameters among the 10 quantumchemical parameters considered (Gaussian software) are most often the total energy (TE) and frontier orbital energy LUMO (E_LUMO). On the other hand, we can distinguish GETAWAY (GEometry, Topology, and Atom-Weights AssemblY) descriptors next to 3D-MoRSE (3D-Molecule Representation of Structures based on Electron diffraction) descriptors from Dragon software, which leads to the assumption that a functional dependency exists. These parameters seem to be particularly important for psychoactive activity and properties of analyzed structures, which is related to the hypotheses regarding the mechanism of action of compounds with this type of elements of structure and mostly their pharmacological classification. It seems advisable also to continue research in the extended database of molecules and their experimental values of pharmacological/physicochemical properties.

\section{Compliance with ethical standards}

Conflict of interest The authors declare that they have no conflict of interest.

Open Access This article is distributed under the terms of the Creative Commons Attribution 4.0 International License (http://crea tivecommons.org/licenses/by/4.0/), which permits use, duplication, adaptation, distribution, and reproduction in any medium or format, as long as you give appropriate credit to the original author(s) and the source, provide a link to the Creative Commons license, and indicate if changes were made.

\section{References}

Cogordan JA, Mayoral M, Angeles E, Toscano RA, Martinez R (1999) Neuroleptic and antidepressant tricyclic compounds: theoretical study for predicting their biological activity by semiempirical, density functional, and Hartree-Fock methods. Int J Quant Chem 71:415-432

Davis PW, Brody TM (1966) Inhibition of $\mathrm{Na}+\mathrm{K}+$-activated adenosine triphosphatase activity in rat brain by substituted phenothiazines. Biochem Pharmacol 15:703-710

Dean CE (2011) Psychopharmacology: a house divided. Prog Neuropsychopharmacol Biol Psychiatry 35:1-10

Dragon 7 molecular descriptors (2018). https://chm.kode-solutions.net/ products_dragon.php. Accessed 12 March 2018

Hendriksen H, Groenink L (2015) Back to the future of psychopharmacology: a perspective on animal models in drug discovery. Eur J Pharmacol 759:30-41

Official Gaussian Website (2018). http://www.gaussian.com/. Accessed 12 March 2018

Nasal A, Buciński A, Bober L, Kaliszan R (1997) Prediction of pharmacological classification by means of chromatographic parameters processed by principal component analysis. Int $\mathrm{J}$ Pharm 159:43-55

Saucin M, van der Vorst A (1972) On the formation of charge transfer complexes between neuroleptic drugs and chloranil. Biochem Pharmacol 21:2673-2680

Spiegel R (2003) Psychopharmacology: an introduction, 4th edn. Wiley-Blackwell, Hoboken

Stahl SM (2013) Stahl's essential psychopharmacology neuroscientific basis and practical applications, 4th edn. Cambridge University Press, Cambridge

Todeschini R, Consonni V (2010) Molecular descriptors for chemoinformatics: volume i: alphabetical listing /volume ii: appendices, references, vol 41. Wiley-VCH Verlag $\mathrm{GmbH} \& \mathrm{Co}$. $\mathrm{KGaA}$, Weinheim

Unger SH, Chiang GH (1981) Octanol-physiological buffer distribution coefficients of lipophilic amines by reversed-phase highperformance liquid chromatography and their correlation with biological activity. J Med Chem 24:262-270 